\title{
First challenge on Lagrangian Particle Tracking and Data Assimilation: datasets description and planned evolution to an open online benchmark
}

\author{
Benjamin Leclaire ${ }^{1, *}$, Ivan Mary ${ }^{2}$, Cédric Liauzun $^{2}$, Stéphanie Péron $^{2}$, Andrea Sciacchitano $^{3}$, Andreas \\ Schröder ${ }^{4}$, Philippe Cornic ${ }^{5}$ and Frédéric Champagnat ${ }^{5}$
}

\author{
1: ONERA, Department of Aerodynamics, Aeroelasticity and Acoustics, Meudon, France \\ 2: ONERA, Department of Aerodynamics, Aeroelasticity and Acoustics, Châtillon, France \\ 3: Delft University of Technology, Faculty of Aerospace Engineering, Delft, The Netherlands \\ 4: DLR, Institute of Aerodynamics and Flow Technology, Göttingen, Germany \\ 5: ONERA, Department of Information Processing and Modelling, Palaiseau, France \\ *corresponding author: benjamin.leclaire@onera.fr
}

In the last decade, Lagrangian Particle Tracking (LPT) has emerged as one of the leading measurement techniques for the quantitative determination of fluid flows in three-dimensional domains (see e.g. Schanz et al., 2016), due to its accuracy in reconstructing particles velocities and material accelerations. Due to the scattered nature of the obtained result, at the particles positions only, significant research efforts have also been placed in the development of dedicated Data Assimilation (DA) techniques, aiming at finally reconstructing full 3D velocity and pressure fields on regular Cartesian grids (see, e.g., Schneiders et al. 2016).

Within the framework of the Horizon 2020 project HOMER (Holistic Optical Metrology for Aero-Elastic Research), research groups at DLR, ONERA and TU Delft have jointly organized the $3^{\text {rd }}$ Workshop and $1^{\text {st }}$ Challenge on DA \& CFD Processing for PIV and LPT. The challenge purpose was to assess the state-of-the-art on LPT and DA algorithms in a situation reflecting a typical experimental campaign. The turbulent wall-bounded flow in the wake of a cylinder was chosen, being a situation with important turbulent fluctuations due to the wake, both in velocity and pressure. Diameter $D=0.01 \mathrm{~m}$ and gap from the wall $G=0.01 \mathrm{~m}$ of the cylinder were chosen in comparison with the targeted boundary layer thickness $\delta \approx 60 \mathrm{~mm}$, and with reference to studies in literature on vortex shedding with ground effect (see e.g. Wang \& Tan 2008), in order to obtain standard shedding behaviour while maintaining large enough wall pressure fluctuations.

Monotone Integrated Large Eddy Simulations (MILES) were performed with the ONERA HPC multi-block structured aerodynamic solver FASTS, using second-order finite volume spatial discretization and second-order implicit time integration. The computational domain size is of $1.8 \mathrm{~m} \times 0.1 \mathrm{~m} \times 1.0 \mathrm{~m}$ in the streamwise $(X)$, spanwise $(Y)$ and wallnormal $(Z)$ directions, with mesh cell number of $1367 \times 500 \times 247=168,824,500$ cells. The flow zone used for the datasets is of $0.1 \mathrm{~m} \times 0.05 \mathrm{~m} \times 0.03 \mathrm{~m}$; it is centred in span, with its upstream end located $0.035 \mathrm{~m}$ downstream of the cylinder centre, and extending from the wall to $0.03 \mathrm{~m}$ above the wall. Typical cell size in this region was $\Delta X=$ $0.4 \mathrm{~mm}, \Delta Y=0.2 \mathrm{~mm}$ and $0.0165 \mathrm{~mm} \leq \Delta Z \leq 0.47 \mathrm{~mm}$. The method of Lund et al. (1998) was used to obtain a developed turbulent boundary layer, and periodicity was imposed in the spanwise direction. Free-stream Reynolds number per unit length was set to $R e_{\infty, x}=665,000$, leading to a boundary layer momentum thickness Reynolds number of $R e_{\theta}=4,150$ ten diameters upstream of the cylinder. From these simulations, similarity was used to transpose the initial air flow situation towards a water flow with similar dimensions, thereby leading to equivalent free-stream velocity $U_{\infty}=0.667 \mathrm{~m} . \mathrm{s}^{-1}$. Figures 1 and 2 respectively present a sample flow snapshot, and the time- and span-averaged mean and fluctuating streamwise velocity component, illustrating the intensity of the turbulent fluctuations, largely due to the shed vortices and their secondary structures. As shown in particular by Figure 2, the flow zone of the dataset corresponds to a progressive wake recovery. Simulations were run with embedded propagation of synthetic pointwise tracer particles, whose positions were initially chosen randomly, using interpolation of the velocity field and a $3^{\text {rd }}$ order Adams-Bashforth time scheme. Overall, 22 sets of particle trajectories of different lengths, each corresponding to different starting flow instants, were generated in order to build all datasets, consisting of two-pulse (TP), four-pulse (FP) or time-resolved (TR) data for the LPT challenge, and of TR data for the DA challenge. Analysis of the trajectories (not shown here) indicated that significant curvatures and accelerations logically coincided mostly with shed vortices, and in a lesser extent with near-wall turbulent structures.

Synthetic particle images for the LPT cases were obtained by considering four virtual cameras with a sensor size of $1920 \times 1200$ pixels and $10 \mu \mathrm{m}$ pitch, using pinhole projection with no Scheimpflug and no distortions, camera locations and focal lengths leading to an equivalent voxel size of $60 \mu \mathrm{m}$. Calibration data (list of points and projections), with no error, were provided to the participants. Particles were given a polydisperse intensity distribution, then spread to form the images using a Gaussian $(\sigma=0.6)$ point-spread function, modelling diffraction-limited imaging. Thermal and shot noise were also added in the images. Datasets were generated corresponding to $0.005,0.025,0.05,0.08,0.12,0.16$ particles per pixel (ppp), for the TP and FP cases, and also to $0.2 \mathrm{ppp}$ for the TR case. The three DA cases (corresponding to $0.005,0.025$ and $0.16 \mathrm{ppp}$ ) were generated by adding a random Gaussian position noise (with $\sigma=0.1$ voxel) to the raw trajectories. 
These datasets were publicly released for download on March $9^{\text {th }}$, 2020, see https://w3.onera.fr/first_lpt and_da challenge/. Participants were then requested to upload their processed results by July $17^{\text {th }}, 2020$, consisting of particles positions and velocities (and possibly accelerations depending on the cases) for the LPT challenge, and of velocity, velocity gradient and pressure fields on a prescribed 3D grid for the DA challenge. Analysis of the participants results will be presented in two separate communications also submitted to the ISPIV2021 symposium (Sciacchitano et al. and Sciacchitano et al.).

Future steps of this work, of which some are underway, are to complete the challenge data portal with an automatic assessment capability, with error and performance metrics computed and communicated to the participant upon data upload, in a similar idea as widely done in the computer vision community (see, e.g. Geiger et al. 2012). Additionally to a full presentation of the flow simulation and final datasets of the LPT and DA challenges, this communication will present the first development steps and principles of this future open online benchmark, which is foreseen to be available to any team interested in development of LPT and DA methods, and conceived to be, in the long run, progressively completed with further datasets relevant to the field.

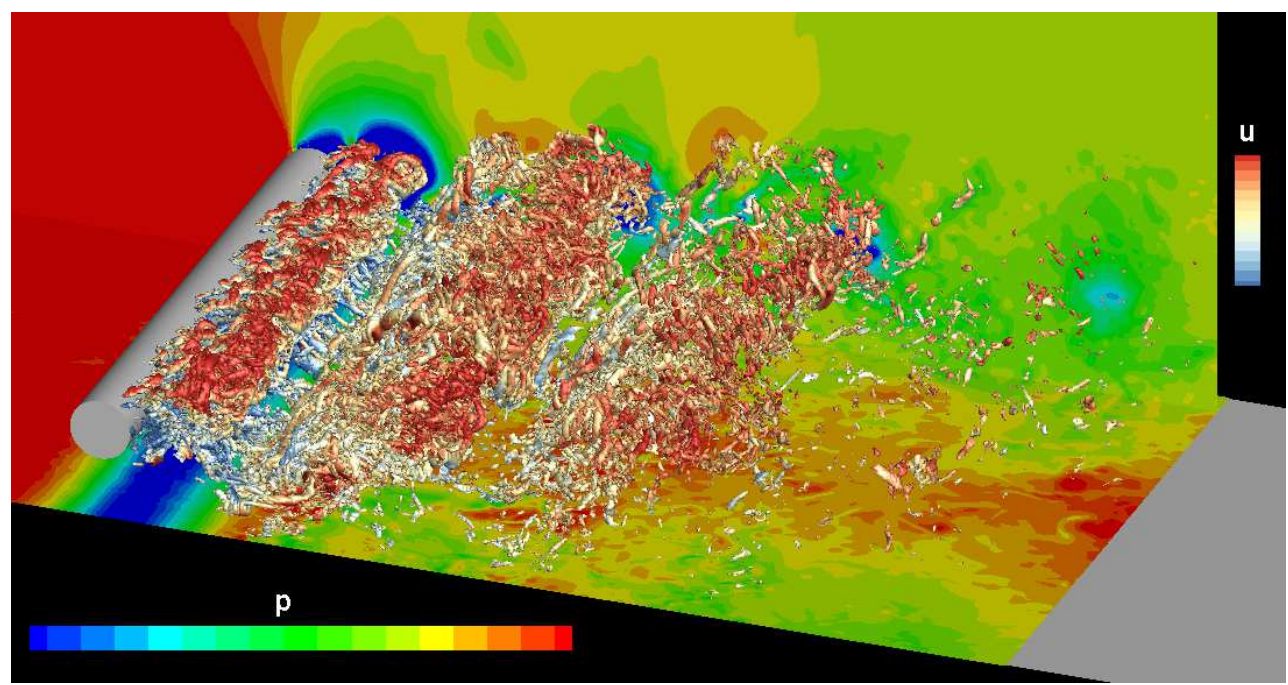

Figure 1- Sample flow snapshot, including Q-criterion iso-surfaces color-coded by streamwise velocity u, isocontours of pressure $p$ at the lower and side walls.
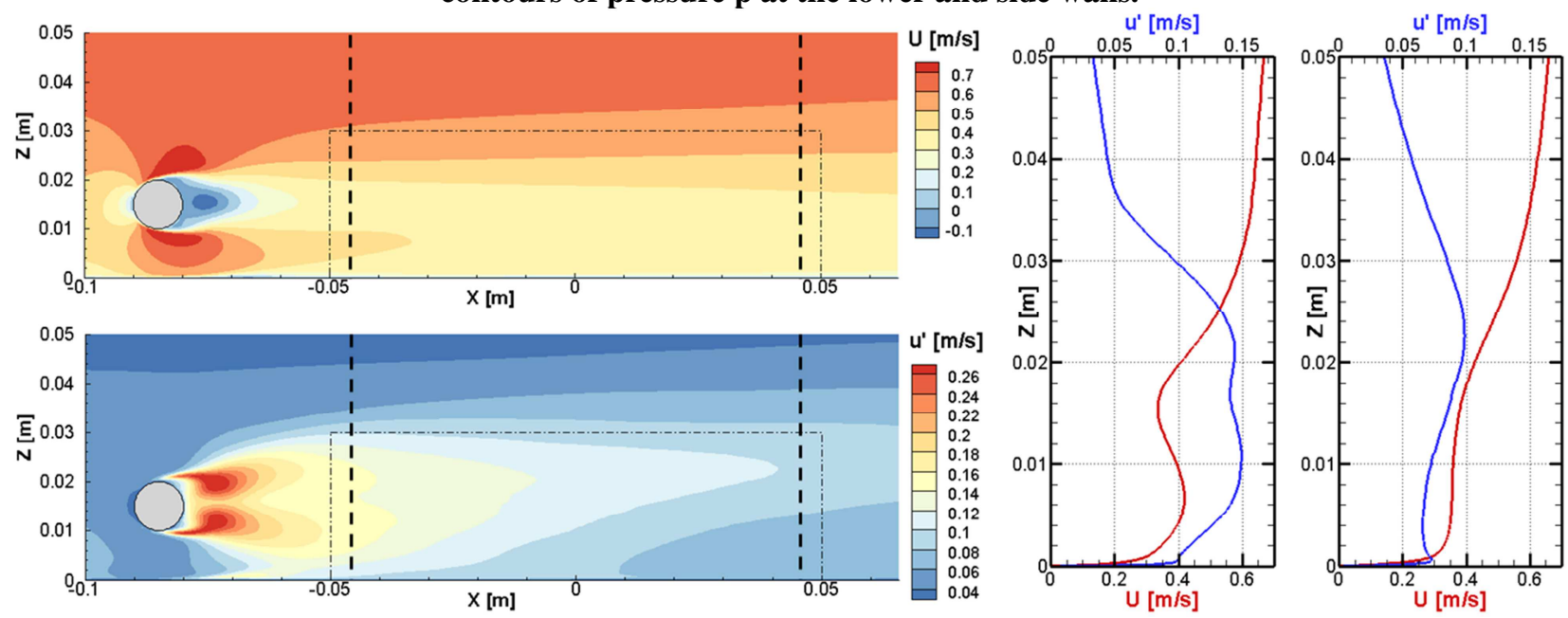

Figure 2- Streamwise mean and fluctuating velocity components, with averaging performed in time and span. Profiles on the right correspond to streamwise locations depicted by the vertical dashed lines in the left figures.

\section{References}

Geiger, A., Lenz, P., \& Urtasun, R. Are we ready for autonomous driving? the kitti vision benchmark suite, 2012, June. In 2012 IEEE Conference on Computer Vision and Pattern Recognition (pp. 3354-3361). IEEE.

Lund, T. S., Wu, X., \& Squires, K. D. Generation of turbulent inflow data for spatially-developing boundary layer simulations. Journal of computational physics, 1998, 140(2):233-258.

Schanz D, Gesemann S, Schröder A. Shake-The-Box: Lagrangian particle tracking at high particle image densities. Experiments in fluids, 2016 May 1;57(5):70

Schneiders JF, Scarano F. Dense velocity reconstruction from tomographic PTV with material derivatives. Experiments in fluids, 2016 Sep 1;57(9):139

Wang, X. K., \& Tan, S. K. Near-wake flow characteristics of a circular cylinder close to a wall, 2008, Journal of Fluids and Structures, 24(5):605-627. 